\title{
R-Process Nucleosynthesis in Alfvén Wave-driven Proto-Neutron Star Winds
}

\author{
Takeru K. Suzuki,; Shigehiro Nagataki, Shinya Wanajo \\ E-mail: stakerueprovence.c.u-tokyo.ac.jp, \\ nagataki@yukawa.kyoto-u.ac.jp, wanajo@astron.s.u-tokyo.ac.jp
}

We propose magnetic proto-neutron star (PNS) winds driven by Alfvén waves as well as the neutrino heating as an appropriate site for the r-process nucleosynthesis. Alfvén waves excited by surface motions of a PNS propagate outwardly, and they heat and accelerate the wind by dissipation. In the Alfvén wave-driven wind, larger entropy per baryon and shorter dynamical time scale are achieved, which favors the r-process. A PNS with surface $B_{0} \gtrsim 5 \times 10^{14} \mathrm{G}$, gives suitable wind properties for the r-process in a typical case. We also perform nuclear reation calcuations and confirm this result; the 3rd peak elements are sufficiently synthesized in the Alfvén wavedriven wind in such a condition.

International Symposium on Nuclear Astrophysics - Nuclei in the Cosmos - IX

June 25-30 2006

CERN, Geneva, Switzerland

\footnotetext{
${ }^{*}$ Gradate School of Arts \& Sciences, University of Tokyo

†Yukawa Institute for Theoretical Physics, Kyoto University; Kavli Institute for Particle Astrophysics and Cosmology Stanford Linear Accelerator Center

$\doteqdot$ Department of Astronomy, University of Tokyo
} 


\section{Introduction}

A neutrino-driven wind is probably the most promising site of the rapid neutron capture process (r-process). However, it is difficult to realize the suitable condition for the r-process in the wind of the standard proto-neutron star (PNS) (e.g. [1]). Recently roles of magnetic fields are highlighted to suitable circumstances for the r-process, namely short dynamical timescale, $t_{\exp }$, and large entropy per baryon, $S$. Thompson (2003) [2] considered large-scale scale magnetic fields which confine the plasma to achieve enough $S$. As another possibility, Suzuki \& Nagataki [3] proposed that Alfvén waves generated from magnetic PNS give faster acceleration (smaller $t_{\exp }$ ) and larger heating (larger $S$ ) in the wind by the additional momentum and energy inputs. In this contribution, we firstly introduce basic properties of Alfvén wave-driven winds, and then, present results of nucleosynthesis calculations.

\section{Model}

We consider steady-state winds from PNSs with radius, $R_{\mathrm{NS}}=10 \mathrm{~km}$, under the Newtonian gravity. We assume radial expansion of magnetic flux tubes in which the winds flow out, giving magnetic flux conservation, $B r^{2}=B_{0} r_{0}^{2}$, where $B_{0}$ is the surface magnetic field strength. Difference from the standard neutrino-driven wind is that we take into account the acceleration by the wave pressure, $P_{w}$, in the momentum equation and the heating, $\dot{q}_{w}$, by the wave dissipation in the energy equation which are

$$
v \frac{d v}{d r}=-\frac{G M}{r^{2}}-\frac{1}{\rho} \frac{d P}{d r}-\frac{1}{\rho} \frac{d P_{w}}{d r}
$$

and

$$
\dot{q_{v}}+\dot{q_{w}}=v\left(\frac{d \mathscr{E}}{d r}-\frac{P}{\rho^{2}} \frac{d \rho}{d r}\right),
$$

respectively, where $\dot{q}_{v}$ is cooling/heating by neutrino and the other variables have the usual meanings. The acceleration $\left(\frac{d P_{w}}{d r}\right)$ and heating $\left(\dot{q}_{w}\right)$ by the Alfvén wave are determined by a suitable dissipation model. Here, We assume wave action, defined as $H_{w} \equiv \frac{\delta B^{2}}{8 \pi} \frac{\left(v_{\mathrm{A}}+v\right)\left(v_{\mathrm{A}}+v\right)}{v_{\mathrm{A}}}$ which is an adiabatic constant in unit of energy flux, follows an exponential decay on $r$ with dissipation length, $l$ :

$$
H_{w}=\frac{R_{\mathrm{NS}}^{2}}{r^{2}} H_{w, 0} \exp \left(\frac{R_{\mathrm{NS}}-r}{l}\right),
$$

We inject Alfvén waves with initial amplitude, $\delta B_{0} / B_{0}=0.1$ at the surface, and we construct transonic wind solutions (see [3] for more detailed setting of the model and subsonic cases. ) We only consider strong magnetic field cases, $B_{0}=10^{14}-10^{15} \mathrm{G}$, because otherwise the Alfvén waves only give a tiny effect on the winds. $l$ can be estimated from the solar wind studies by one of the authors [4-6], in which weakly nonlinear $\left(\delta B_{0} / B_{0} \sim 0.1\right)$ waves dissipate typically after propagating $\sim 10$ wavelengths, corresponding to $l \lesssim 10 R_{\mathrm{NS}}$ in the present PNS conditions.

\section{Results}

Figure 1 shows the structures of the Alfvén wave-driven winds. The left panels present dependence on surface magnetic field, $B_{0}$, and the right panels present dependence on dissipation length, 

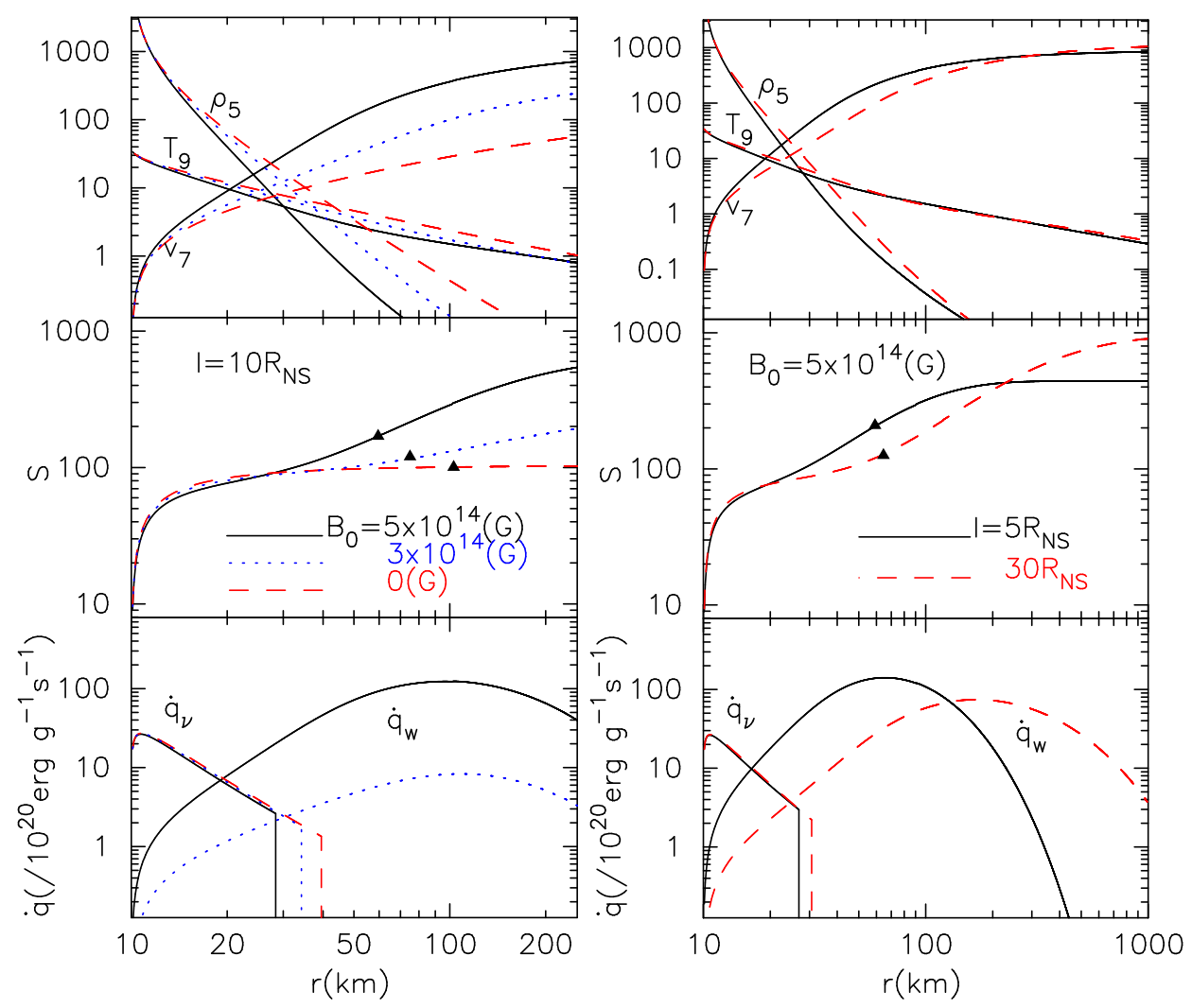

Figure 1: Structures of Alfvén wave-driven winds. The top panels show density in unit of $10^{5} \mathrm{~g} \mathrm{~cm}^{-3}, \rho_{5}$, temperature in $10^{9} \mathrm{~K}, T_{9}$, and velocity in $10^{7} \mathrm{~cm} \mathrm{~s}^{-1}, v_{7}$. The middle panels exhibit entropy per baryon, $S$. The bottom panels show the heating by waves, $\dot{q}_{w}$, and by neutrinos, $\dot{q}_{v}$. The left panels show dependence on $B_{0}\left(=5 \times 10^{14} \mathrm{G}\right.$; solid, $3 \times 10^{14} \mathrm{G}$; dotted, $0 \mathrm{G}$; dashed $)$ for fixed $l=10 R_{\mathrm{NS}}$ and the right panels show dependence on $l\left(=5 R_{\mathrm{NS}}\right.$; solid, $30 R_{\mathrm{NS}}$; dashed) for fixed $B_{0}=5 \times 10^{14} \mathrm{G}$.

l. Thanks to the wave pressure, the wind is accelerated faster as $B_{0}$ increases. The heating due to the wave dissipation also gives larger $S$ in the larger $B_{0}$ case. Therefore, the conditions in the Alfvén wave-driven winds are favorable for the r-process. Faster dissipation (smaller $l$ ) leads to more rapid increase of $S$, which is also better for the r-process.

In Figure 2 we compare the results of $t_{\exp }$ and $S$ with the r-process condition ${ }^{1}$ by [1],

$$
S \gtrsim 2 \times 10^{3} Y_{e}\left(\frac{t_{\mathrm{exp}}}{s}\right)^{1 / 3}
$$

where $Y_{e}$ is electron fraction which our model does not explicitly include. For standard $Y_{e}(=0.4-$ 0.5 ), Alfvén -driven winds of PNSs with $B_{0} \gtrsim 5 \times 10^{14} \mathrm{G}$ satisfy the condition, provided $l<$ $10 R_{\mathrm{NS}}$.

Next, we calculate the actual nucleosynthesis in the Alfvén wave-driven winds [8]. We perform nuclear reaction network calculations by adopting the physical conditions $(\rho$ and $T$ ) which change with time according to the outward velocities along with the flows. Figures 3 and 4 show the dependences of the synthesized elements on $B_{0}$ and $l$, where we assume electron fraction $=0.4$.

\footnotetext{
${ }^{1}$ This is strictly the condition for $\alpha$-process [7] which determines a neutron-to-seed ratio before the neutron capture occurs.
} 


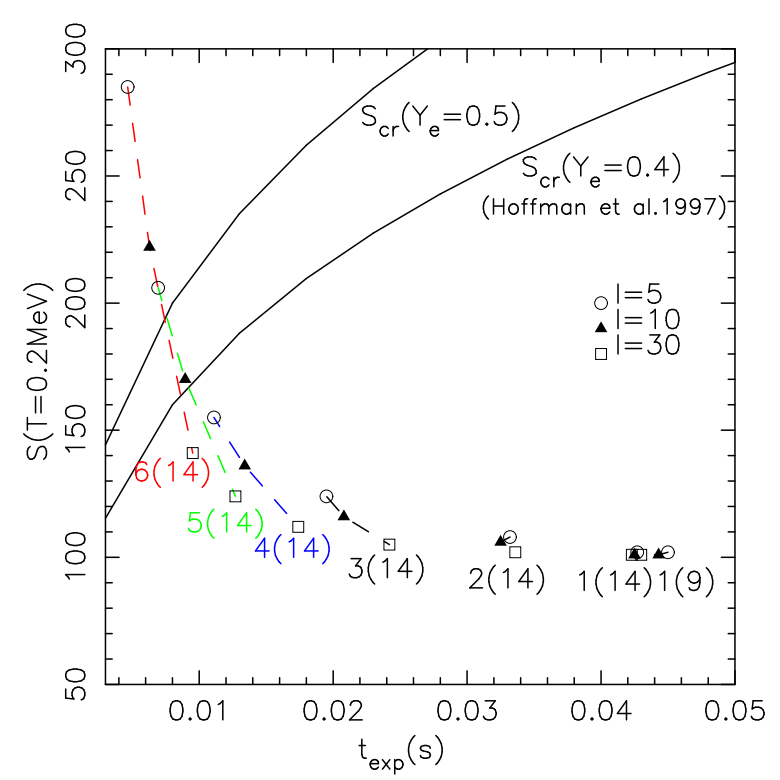

Figure 2: $t_{\exp }(\mathrm{s})(\mathrm{X}$-axis) and $S$ at $T=0.2 \mathrm{MeV}$ (Y-axis) for various parameters of Alfvén waves. Open circles, filled triangles, and open squares are results with $l=5,10, \& 30$, respectively. Numbers denote magnetic field strength at the surface. For example 6(14) indicates $B_{0}=6 \times 10^{14} \mathrm{G}$. Results with the same $B_{0}$ are connected by dashed lines. Solid lines are the conditions for the r-process by [1] for $Y_{e}=0.4 \& 0.5$.

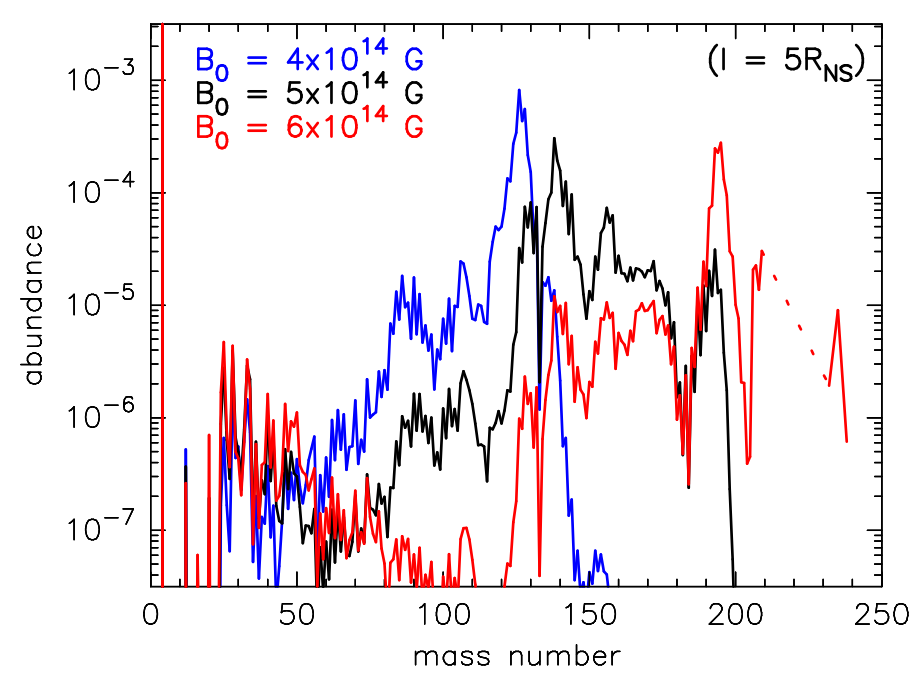

Figure 3: Results of the synthesized elements in the Alfvén wave-driven winds. The red, black, and blue lines correspond to $B_{0}=6 \times 10^{14} \mathrm{G}, 5 \times 10^{14} \mathrm{G}$, and $4 \times 10^{14} \mathrm{G}$, respectively, where we adopt constant $l=5 R_{\mathrm{NS}}$. 


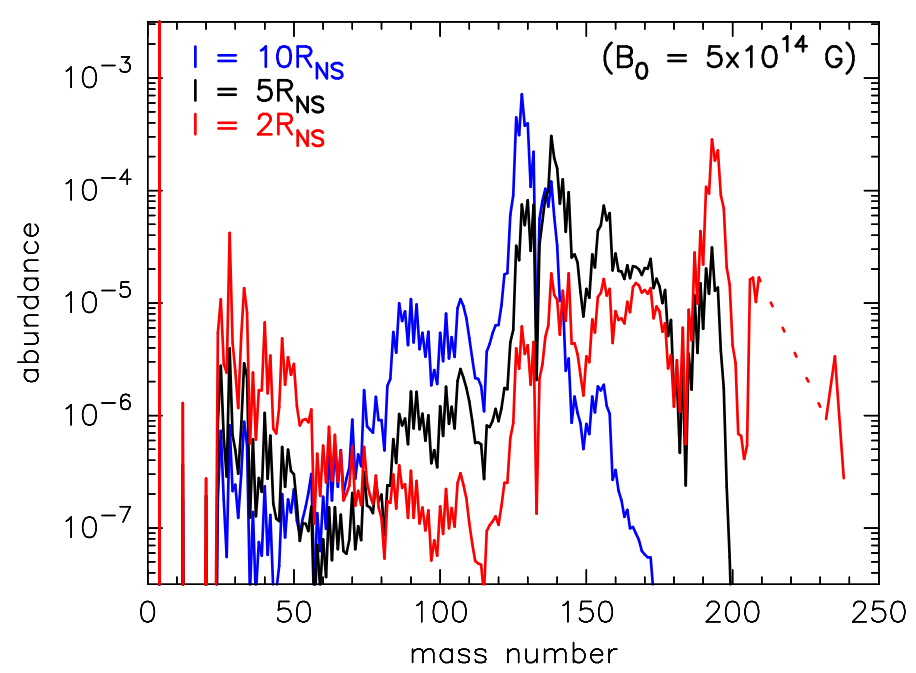

Figure 4: The same as Figure 3 but for the $\nmid$ dependence. The red, black, and blue lines correspond to $l=2 R_{\mathrm{NS}}, 5 R_{\mathrm{NS}}$, and $10 R_{\mathrm{NS}}$, respectively, for $B_{0}=5 \times 10^{14} \mathrm{G}$.

Figure 3 illustrates that larger $B_{0}$ favors the r-process. While the 3 rd peak elements (mass number, $A \approx 195$ ) are not produced in the $B_{0}=4 \times 10^{14} \mathrm{G}$ case (see [8] for non magnetic field case), they are synthesized in the $B_{0}=(5,6) \times 10^{14} \mathrm{G}$ cases. In the $B_{0}=6 \times 10^{14} \mathrm{G}$ case, sizable amounts of elements with $A=230-240$ are also synthesized. This is because the nonequilibrium circumstances with small $t_{\text {exp }}$ and large $S$ are achieved in larger $B_{0}$, which is suitable for the $\mathrm{r}$ process.

Figure 4 exhibits that smaller $l$ is favorable to the synthesis of the r-process elements; the 3 rd peak elements are produced in the cases with $l=(2,5) R_{\mathrm{NS}}$, while they are not in the case with $10 R_{\mathrm{NS}}$. This is because $S$ should be large in the inner region where the $\alpha$-process [7] takes place to give a sufficiently large neutron-to-seed ratio, which requires small $l$. Otherwise if $l$ is large, the wave heating occurs in the outer region and the increase of $S$ is a little to slow; in the case with $l=10 R_{\mathrm{NS}}$, the 3 rd peak elements are not synthesized.

\section{References}

[1] Hoffman, R. D., Woosley, S. E., \& Qian, Y.-Z. 1997, ApJ, 482, 951

[2] Thompson, T. A. 2003, ApJ, 585, L33

[3] Suzuki, T. K. \& Nagataki, S. 2005, ApJ, 628, 914

[4] Suzuki, T. K. 2004, MNRAS, 349, 1227

[5] Suzuki, T. K. \& Inutsuka, S. 2005, ApJ, 632, L49

[6] Suzuki, T. K. \& Inutsuka, S. 2006, JGR, 111, A06101

[7] Woosley, S. E. \& Hoffman, R. D. 1992, ApJ, 395, 202

[8] Wanajo, S., Suzuki, T. K. \& Nagataki, S. 2006, submitted to ApJL 